\title{
Lumbar nephroblastoma in a young crossbreed dog
}

\author{
Max Foreman*, Roberta Rasotto \& Salih Eminaga \\ Dick White Referrals $•$ Station Farm $・$ London Road $\bullet$ Six Mile Bottom $・$ Suffolk CB8 0UH $・$ United Kingdom \\ *max.foreman@dwr.co.uk \\ Introduction and Objectives
}

Spinal cord nephroblastomas are embryonal tumors that are thought to arise from embryonic renal tissue that becomes trapped in the dura mater during fetal development, and later in life undergoes neoplastic transformation. ${ }^{1}$ Spinal cord nephroblastomas are typically intradural-extramedullary tumors, and in dogs most commonly cause clinical signs between 0.5 - 4 years of age, with neurological deficits consistent with T3-L3 myelopathy. Reports of spinal cord nephroblastomas located outside of the T3-L3 segments are very rare. ${ }^{2}$

To describe the presenting signs, imaging findings and histopathological confirmation of a spinal nephroblastoma in a young crossbreed dog.

\section{Case Presentation}

A 1-year-old male neutered Collie-cross dog was examined for evaluation of a three-week history of right pelvic limb lameness progressing to pelvic limb weakness as well as urinary and faecal incontinence. Radiography of the lumbar spine, pelvis, coxofemoral joints and stifles had documented the presence of eight lumbar vertebrae but were otherwise unremarkable. General physical examination was unremarkable.

Neuroanatomical Localisation: L4-S3 Spinal Cord Segments (left-lateralised)
- Mentation: Bright, alert, responsive, appropriate

- Gait: Ambulatory paraparesis without ataxia

- Urinary and faecal incontinence when walking

- Posture: Reduced tail tone

- Cranial Nerve Exam: Normal

- Proprioception: Reduced both pelvic limbs

- Segmental Spinal Reflexes: Reduced-absent left pelvic limb (withdrawal and patella). Perineal reflex absent.

- Palpation: Lumbar hyperaesthesia. Reduced anal tone. Large flaccid urinary bladder, easily expressed ('lower motor neuron bladder').

\section{Results}

Serum biochemistry and haematology were largely unremarkable. Magnetic resonance imaging (Fig. 1) showed a T1 and T2 hyperintense (to spinal cord), ovoid, broad-based intradural extramedullary mass lesion extending along the length of the vertebral body of L5. There was moderate homogenous contrast enhancement of the mass, measuring $2.1 \times 1.0 \times 1.1 \mathrm{~cm}$. The owner elected for euthanasia. Post-mortem histopathological examination (Fig. 2) of the mass confirmed an intradural mass compressing and multifocally infiltrating the nerve roots and spinal cord. The mass was encapsulated and composed of a disorganized mixture of epithelial, blastemal and mesenchymal cells. The mass was considered to have unequivocal features of an ectopic spinal nephroblastoma. ${ }^{3}$
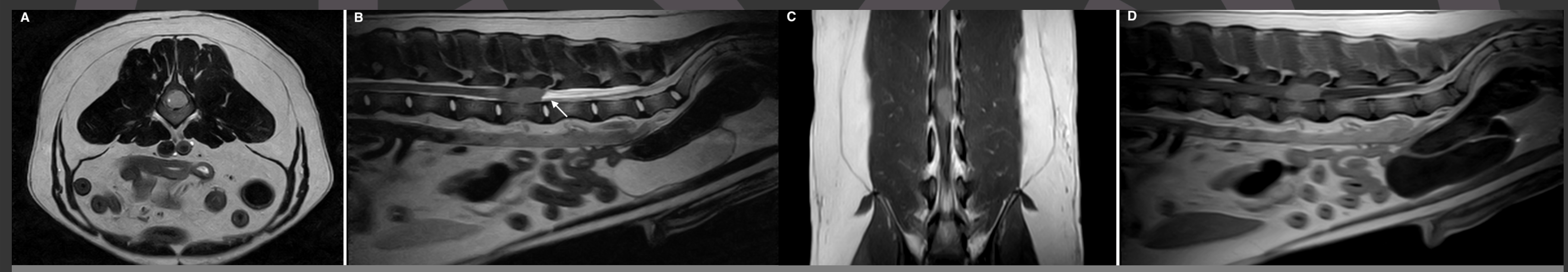

Figure 1 (above): MRI images. A-Transverse T2w image at the level of L5 - the lesion is hyperintense and occupies almost the entire cross section of the spinal canal. The spinal cord is displaced to the right with severe compression. B-Sagittal T2w image - the lesion has an ovoid and broad-based appearance; notice a so-called 'golf-tee sign' (arrow), characteristic of an intradural extramedullary location. C-Dorsal $\mathrm{T} 1 \mathrm{w}+\mathrm{C}$ image demonstrating moderate homogeneous contrast enhancement of the lesion with no visible extension into the surrounding vertebral column. The lesion appears to be extramedullary. D-Sagittal T1w+C - some degree of cranial intramedullary extension is difficult to fully exclude.

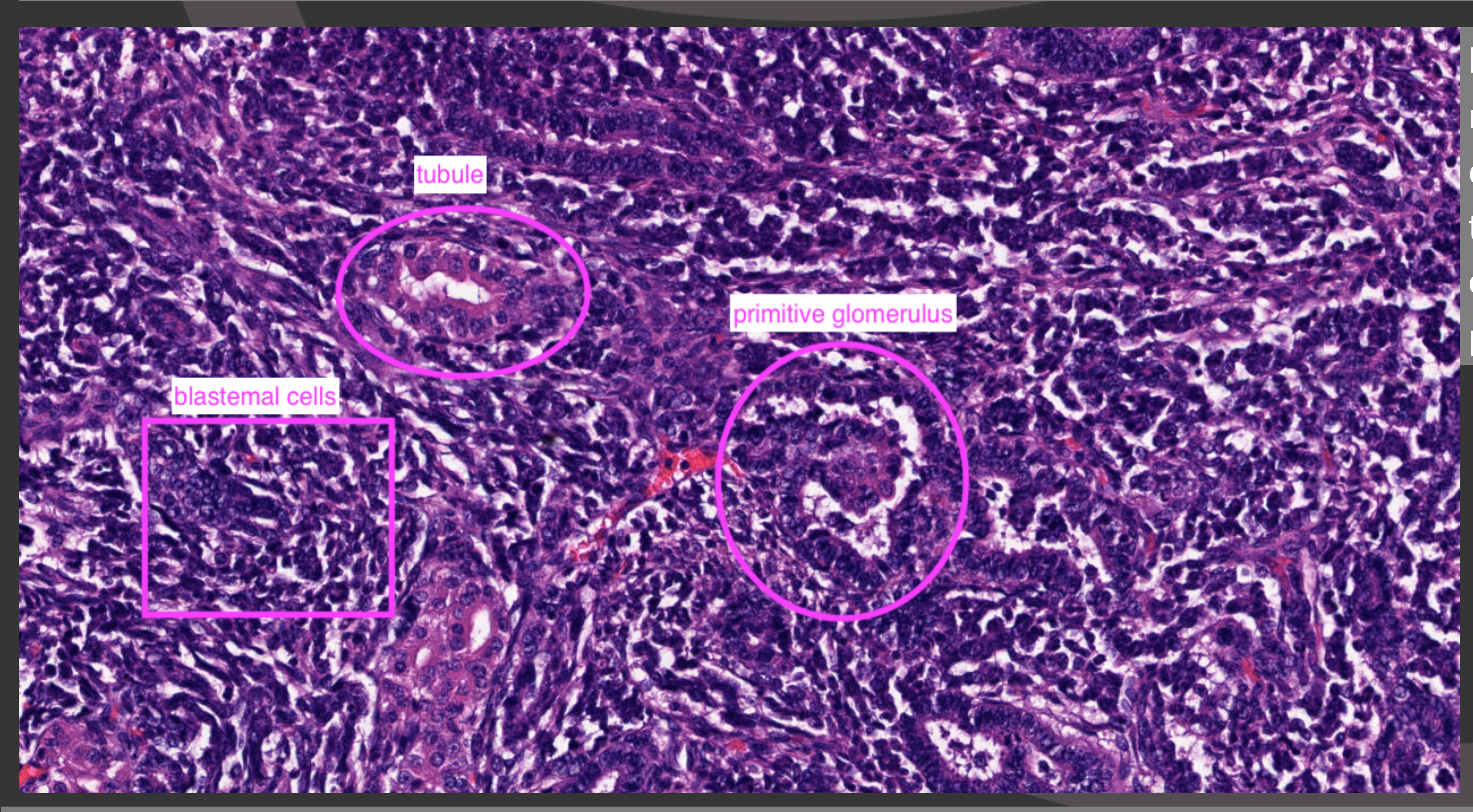

Figure 2 (left): Histological photomicrograph (haematoxylin and eosin stain, 20x magnification), demonstrating two of the three neoplastic cell populations (epithelial cells and blastemal cells). Epithelial cells were cuboid to columnar and arranged in tubules and occasional primitive glomeruli. Blastemal cells were roundish to occasionally polygonal with high nuclear/cytoplasmic ratio and formation of patternless sheets.

\section{References}

1 Sale CS, Skerritt GC, Smith KC. Spinal nephroblastoma in a crossbreed dog. J Small Anim Pract 2004;45:267-271.

2 Brewer DM, Cerda-Gonzalez S, Dewey CW, et al. Spinal cord nephroblastoma in dogs: 11 cases (1985-2007). J Am Vet Med Assoc 2011;238:618-624.

3 De Lorenzi D, Baroni M, Mandara MT. A true "triphasic" pattern: thoracolumbar spinal tumor in a young dog. Vet Clin Pathol 2007;36:200-203. 